ISSN 1997-5902

\title{
Statut des principales maladies cryptogamiques foliaires du blé au Maroc en 2013
}

\author{
Samir ZAHRI, Ali FARIH et Allal DOUIRA \\ Laboratoire de Botanique et de Protection des Plantes, Université Ibn Tofaïl, Faculté des Sciences, B.P. 133, Kénitra, \\ Maroc. \\ Laboratoire de Phytopathologie, Département de Phytiatrie, INRA, El Menzeh, Kénitra, B.P. 293, Maroc. \\ Emails: zahri_samir@yahoo.fr / douiraallal@hotmail.com
}

Original submitted in on 21st December 2013. Published online at www.m.elewa.org on $31^{\text {st }}$ May 2014. http://dx.doi.org/10.4314/jab.v77i1.5

\section{RÉSUMÉ}

Objectif : Cette étude décrit le statut des principales maladies cryptogamiques foliaires du blé dans 3 régions céréalières du Nord-Ouest Marocain durant la campagne céréalière 2012-2013. En effet, un inventaire de ces maladies a été établi.

Méthodes et résultats : Cinquante (50) champs de blé tendre et quarante deux (42) champs de blé dur ont été prospectés en avril-mai 2013, au moment de la floraison et de la maturation de la culture. Les maladies ont été identifiées sur la base de leurs symptômes typiques et l'observation microscopique des spores des pathogènes après incubation des échantillons. Les prévalences, incidences et sévérités d'attaques ont été déterminées pour chaque maladie cryptogamique foliaire. Les septorioses, les rouilles brunes et les helminthosporioses étaient de loin les maladies foliaires les plus importantes dans les champs de blé tendre, avec des prévalences de $85,4,76,6$, et $63,0 \%$ respectivement. Au niveau des champs de blé dur, la situation a été caractérisée par la prédominance de la rouille brune $(79,0 \%)$, l'helminthosporiose $(70,1 \%)$, et les septorioses $(45,8 \%)$. L'oïdium était le moins prévalent (14,2\%) sur le blé tendre et $11,4 \%$ sur le blé dur et le moins sévère $(8,0 \%)$ vu son apparition tardive dans le cycle du blé. Aucun symptôme des rouilles jaunes ou noires n'a été révélé dans cette étude.

Conclusion et application : Malgré la production céréalière record de la campagne 2012-2013 (plus de 97 millions de quintaux) due à une pluviométrie exceptionnelle, ces conditions climatiques ont potentialisé le développement et la propagation des maladies cryptogamiques. Suite à cette situation inquiétante, et pour atténuer leur impact sur le rendement, un programme de traitements fongicides a été généralisé. La septoriose due à Septoria tritici a été la plus dominante parmi les maladies identifiées.

Mots clés : Maroc, blé, statut, inventaire, maladies cryptogamiques, prévalences, incidences, sévérités.

\begin{abstract}
.
Objective: This study describes the status of the principal cryptogamic foliar diseases of wheat in three cereal regions in the northwest of Morocco during the 2012-2013 season cereal. An inventory of these diseases has been established.

Methods and results: Fifty (50) fields of wheat and forty two (42) durum wheat fields were surveyed between April and May 2013. The diseases were identified based on their typical symptoms and the microscopic examination of the spores of their different fructification after the samples incubation. The prevalence, impact and severity of attacks was determined for each foliar fungal disease. Septoria leaf blotch, brown rust and net
\end{abstract}



Maroc en 2013

blotch were by far the most important foliar diseases in wheat fields, with prevalence of $85.4,76.6$ and $63.0 \%$ respectively. At fields of durum wheat, the situation was characterized by the predominance of brown rust $(79.0$ $\%)$, leaf blight $(70,1 \%)$, and Septoria (45.8\%). Powdery mildew was less prevalent $(14,2 \%)$ on bread wheat and $11,4 \%$ for durum wheat and less severe $(8,0 \%)$ saw its late appearance in the cycle of wheat. No symptoms of yellow or black rust was found in this study.

Conclusion and application: Despite of the record cereal production of the 2012-2013 season (over 97 million $\mathrm{cwt}$ ) due to exceptional rainfall, these climatic conditions have boosted the development and spread of fungal diseases. Following this alarming situation, and to mitigate their impact on the performance, a fungicides program was widespread. Septoria diseases caused by Septoria tritici was the dominant among the identified diseases.

Keywords: Morocco, wheat, status, inventory, fungal diseases, prevalence, impact, severity.

\section{INTRODUCTION}

Le blé est l'une des principales cultures qui nourrit le monde. La production mondiale du blé a été de 650,9 millions de tonnes en 2009 (Boulif, 2011). En 2013, le Maroc a atteint une autre récolte céréalière record avec plus de 97 millions de quintaux, après celle de 2009 avec 102 millions de quintaux. Ce niveau de production exceptionnel est dû à une pluviométrie abondante et généralisée qui a été supérieure à la moyenne (>30\%) avoisinant en cumul les $551 \mathrm{~mm}$ (Balaghi et al., 2013). La superficie moyenne emblavée annuellement en blé avoisine les 3 Millions d'hectares ( 2 millions d'ha de blé tendre et 1 million de blé dur) localisés essentiellement dans le bour favorable $(P>300 \mathrm{~mm})$ (Balaghi et al., 2013). Au Maroc, le blé est sujet à de nombreuses contraintes biotiques, notamment les maladies cryptogamiques qui occasionnent des pertes substantielles aussi bien en rendement qu'en qualité des grains, en conditions environnementales favorables pour l'hôte (pathogène), et quand les variétés utilisées sont sensibles. Le développement de ces maladies est favorisé par les méthodes culturales pratiquées : date de semis précoce, forte dose de semis par hectare, fumure excessive ou insuffisante, monoculture intensive, débris et restes

\section{MATÉRIEL ET MÉTHODES}

Afin d'évaluer l'importance des maladies cryptogamiques de blés, trois prospections ont été organisées en avril-mai 2013 dans 3 régions céréalières du Nord-Ouest Marocain : Gharb (Kénitra, Sidi Slimane, Sidi Kacem et alentours), Zemour-Zaër (Khémisset, Rabat et alentours) et Tangérois (Ouezzane, Chefchaouen et alentours). Ces régions requièrent le moyen le plus élevé des des cultures, et variétés sensibles (Eyal et al., 1987). Les maladies cryptogamiques attaquant le blé peuvent être classées en trois groupes: les maladies telluriques causées par des champignons habitant le sol (fontes de semis, pourritures racinaires et piétin échaudage), les maladies transmises par les semences notamment les caries, les charbons et les maladies de l'épi et les maladies foliaires qui sont évidemment les plus importantes parce qu'elles détruisent les tissus des feuilles dont elles réduisent le rendement photosynthétique (Boulif, 2011). Les principales maladies foliaires du blé au Maroc sont les septorioses, les rouilles brunes et la maladie de la tache bronzée ou helminthosporienne. II est à signaler que l'oïdium ou blanc peut aussi prendre de l'ampleur dans certaines situations, malgré son apparition généralement tardive (Ezzahiri, 2001). Toutes ces maladies sont à caractère explosif et peuvent se propager très rapidement sur les variétés sensibles, lorsque les conditions climatiques leur sont favorables (Ezzahiri, 2001). Cette étude a été réalisée dans le but d'inventorier et d'étudier l'importance et l'évolution des principales maladies cryptogamiques de blés au cours de la campagne agricole 2012-2013.

précipitations ( $P>500 \mathrm{~mm}$ en 2013) et les maladies s'y développent au maximum. Ces prospections ont duré 2 jours chacune, portant sur 50 champs de blé tendre et 42 champs de blé dur semés en 2012 avant l'hiver. Les prospections ont été réalisées au moment où les stades phénologiques des blés s'étalaient de la floraison à la maturité. Au cours de ces prospections, des arrêts le long 
des axes routiers ont été effectués régulièrement tous les 10 à $20 \mathrm{~km}$ à travers les 3 régions céréalières.

Pour chaque champ de blé, dix pieds ont été inspectés au hasard pour la présence de différentes maladies cryptogamiques foliaires. Les prévalences (pourcentage de champs contaminés par une maladie par rapport au nombre de champs prospectés), leurs incidences (pourcentage de plantes attaquées) et leurs sévérités (exprimées en pourcentage de surface foliaire attaquée selon des échelles de notation appropriées à chaque

\section{RÉSULTATS}

Lors des prospections réalisées au courant des mois Avril-Mai 2013, 50 champs de blé tendre et 42 de blé dur ont été examinés dans les principales régions céréalières du Nord-Ouest du Maroc.

Inventaire des maladies associées aux blés: Les maladies cryptogamiques foliaires les plus fréquemment rencontrées dans les champs de blé tendre, sont par ordre d'importance les septorioses (Septoria tritici (Desm.) Rob. et Stagonospora nodorum (Berk.) Castell. \& Germano), les rouilles brunes (Puccinia triticina Erikss), la maladie) ont été déterminées. Les maladies cryptogamiques ont été diagnostiquées et identifiées sur la base de leurs symptômes typiques (Zillinsky, 1983), et sur l'observation microscopique des spores, après désinfection des surfaces (solution de 1 pourcent d'hypochlorite de sodium pendant 1 à 5 minutes) et incubation des fragments de feuilles présentant des symptômes 24 à 48 heures à la température ambiante. La distinction entre les pathogènes est réalisée sur la base de leurs caractéristiques morphologiques.

tache bronzée (Pyrenophora tritici-repentis (Died.) Drechs. (anamorphe Drechslera tritici-repentis (Died.) et à un degré moindre l'oïdium ou blanc (Blumeria (Erysiphe) graminis f.sp. tritici) (tableau 1). Au niveau des champs de blé dur, la situation a été caractérisée par la prédominance de la rouille brune, la tache bronzée, et les septorioses. L'oïdium a été moins important. Sur les deux espèces du blé, les symptômes des rouilles noires et jaunes n'ont pas été identifiés.

Tableau 1. Prévalences (\%) des principales maladies cryptogamiques foliaires du blé dans trois régions céréalières du Maroc lors de la campagne 2012-2013.

\begin{tabular}{l|c|c|c|c|c|c}
\hline \multirow{2}{*}{} & \multicolumn{6}{c}{ \% de champs infectés par régions } \\
\cline { 2 - 8 } Maladies & \multicolumn{2}{|c|}{ Gharb } & \multicolumn{2}{c}{ Zemour Zaer } & \multicolumn{2}{c}{ Tangérois } \\
\hline Septoriose & B. T & B. D & B. T & B. D & B. T & B. D \\
\hline Rouille brune & 74.0 & 35.6 & 69.2 & 38.1 & 85.4 & 45.8 \\
\hline Tache bronzée & 62.5 & 77.5 & 65.1 & 69.4 & 76.6 & 79.0 \\
\hline Oïdium & 50.0 & 70.1 & 56.3 & 68.0 & 63.0 & 66.4 \\
\hline Rouille jaune & 14.2 & 11.4 & 9.2 & 6.4 & 7.1 & 11.3 \\
\hline Rouille Noire & - & - & - & - & - & - \\
\hline B. : & - & - & - & - & - & - \\
\hline
\end{tabular}

B.T : Blé tendre. B.D : Blé dur. - : La maladie n'a pas été observée dans les champs visités.

Les symptômes des principales maladies cryptogamiques foliaires du blé sont représentés dans les figures $1,2,3$ et 4. L'analyse de la variance des prévalences des maladies a montré des différences significatives entre les trois régions et les deux types du blé (tableau 2) 


\section{Zahri et al. J. Appl. Biosci. 2014. Statut des principales maladies cryptogamiques foliaires du blé au}

Maroc en 2013

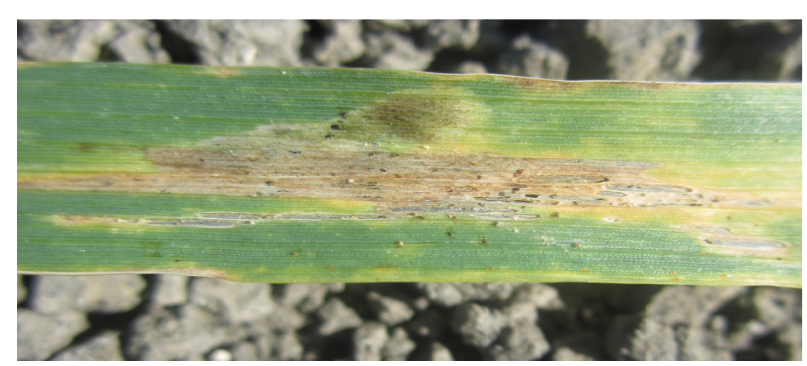

Fig.1: Symptômes de la tache septorienne due à Septoria tritici (photo: S. Zahri)

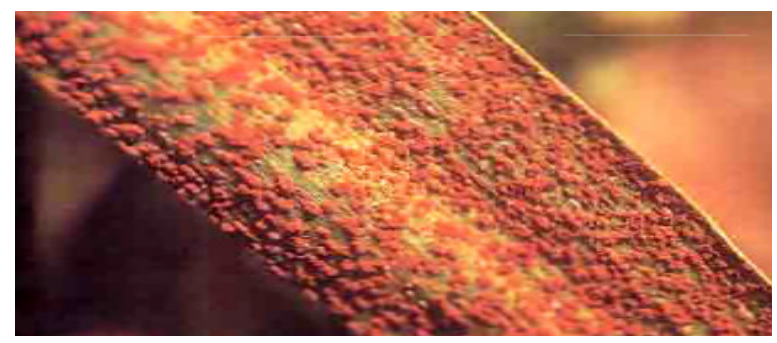

Fig. 2 : Symptômes de la rouille brune due à Puccinia triticina (photo: S. Zahri)

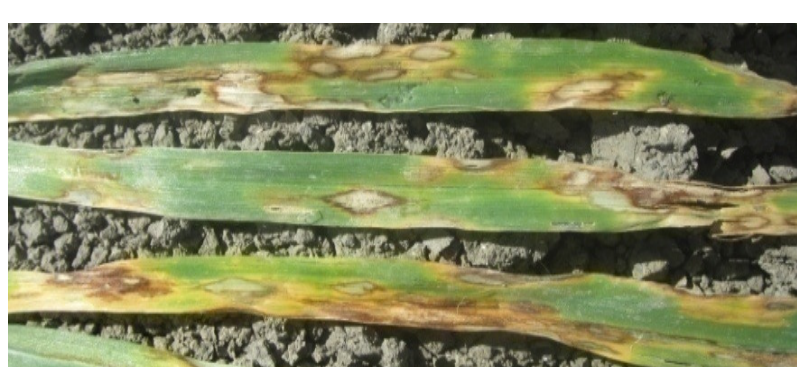

Fig. 3 : Symptômes de la tache bronzée due à $P$. tritici repentis (photo: S. Zahri)

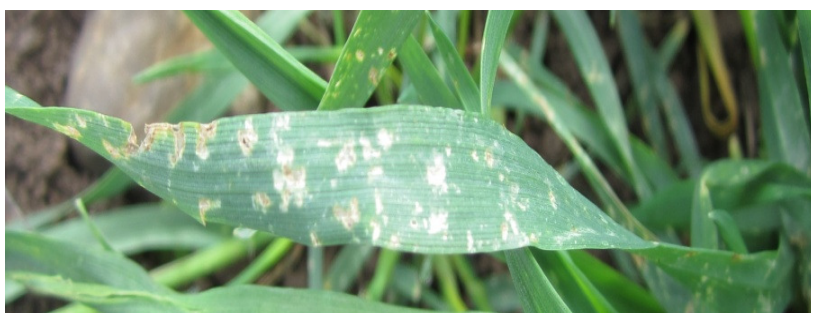

Fig. 4 : Symptômes de l'oïdium due à Erysiphe graminis (photo: S. Zahri)

Tableau 2. Analyse de la variance des prévalences (\%) des principales maladies cryptogamiques foliaires du blé.

\begin{tabular}{|c|c|c|c|c|c|c|}
\hline & \multicolumn{2}{|c|}{ Gharb } & \multicolumn{2}{c|}{ Zemour-Zaer } & \multicolumn{2}{c|}{ Tangérois } \\
\hline Maladies & B. T & B. D & B. T & B. D & B. T & B. D \\
\hline Septoriose & $74.0 \pm 0.91^{*}$ & $35.6 \pm 1.36$ & $69.2 \pm 2.19^{*}$ & $38.1 \pm 0.59$ & $85.4 \pm 1.03^{*}$ & $45.8 \pm 0.30^{*}$ \\
\hline Rouille brune & $62.5 \pm 4.19$ & $77.5 \pm 0.49^{*}$ & $65.1 \pm 2.19^{*}$ & $69.4 \pm 0.37$ & $76.6 \pm 1.13^{*}$ & $79.0 \pm 1.07^{*}$ \\
\hline Tache bronzée & $50.0 \pm 1.85$ & $70.1 \pm 0.74^{*}$ & $56.3 \pm 2.79^{*}$ & $68.0 \pm 0.40^{*}$ & $63.0 \pm 4.43$ & $66.4 \pm 0.55^{*}$ \\
\hline Oïdium & $14.2 \pm 0.76^{*}$ & $11.4 \pm 0.37$ & $9.2 \pm 0.80^{*}$ & $6.4 \pm 0.28^{*}$ & $7.1 \pm 0.30^{*}$ & $11.3 \pm 0.37^{*}$ \\
\hline
\end{tabular}

* La différence moyenne est significative au niveau 0.05 par rapport des autres régions par le test Tukey

La septoriose était présente dans toutes les régions prospectées avec une prédominance bien marquée sur le blé tendre. Sa prévalence était plus élevée sur le blé tendre $(85,4 \%)$ au niveau du Tangérois. Sur le blé dur, les prévalences étaient moins élevées (45, $8 \%$ ) dans la même région. Son incidence moyenne sur le blé tendre était plus importante. Elle fluctue entre 50 et $85 \%$ pour les 50 champs de blé tendre et de 35 à $68 \%$ pour les 42 champs de blé dur. A l'échelle de certaines parcelles, l'incidence de la maladie était de $100 \%$. La maladie était plus sévère sur le blé tendre (70\% Tangérois) que sur le blé dur (30\% Zemour Zaer) (Figure 5). Contrairement à la septoriose, la rouille brune était régulièrement distribuée dans les trois régions prospectées. Ses prévalences étaient plus ou moins similaires sur les deux espèces du blé et varient entre $62,5 \%$ au Gharb et 79,0 $\%$ au Tangérois respectivement sur le blé tendre et dur.
Les incidences étaient assez élevées et fluctuent entre 40 et $75 \%$ pour le blé tendre et entre 38 à $65 \%$ pour le blé dur. La maladie était plus sévère au Gharb pour les blés tendres et durs (48 et $56 \%$ ). Les maladies de rouilles jaunes et noires n'ont pas été observées dans tous les champs visités. La tache bronzée semble être bien distribuée sur blé dur avec des sévérités d'attaques moyennes dans les régions du Gharb (30\%) et Tangérois (42\%). Sur blé tendre, les sévérités étaient moins importantes et varient entre 8 et $18 \%$. La prévalence de la maladie était plus élevée au Gharb $(70,1$ $\%$ ) sur blé dur, plus ou moins similaire des autres régions et au Tangérois (63\%) sur blé tendre. Les incidences moyennes ont été plus élevées sur le blé dur. Elles varient entre 30 et $68 \%$ sur blé dur et entre 18 et $47 \%$ sur blé tendre. L'oïdium était présent dans toutes les régions prospectées mais moins sévère, surtout au 
niveau du Tangérois $(6,5 \%)$ et Zemour - Zaer $(8,0 \%)$. II était plus prévalent au Gharb (14,2\%). Son incidence moyenne était la plus faible par rapport aux autres maladies, variant entre 0 et $35,0 \%$ à l'échelle de la parcelle et entre 13,5 et $21,2 \%$ au niveau régional. Les prévalences, les incidences et les sévérités des principales maladies cryptogamiques foliaires de blés sont illustrées dans la figure 5 .

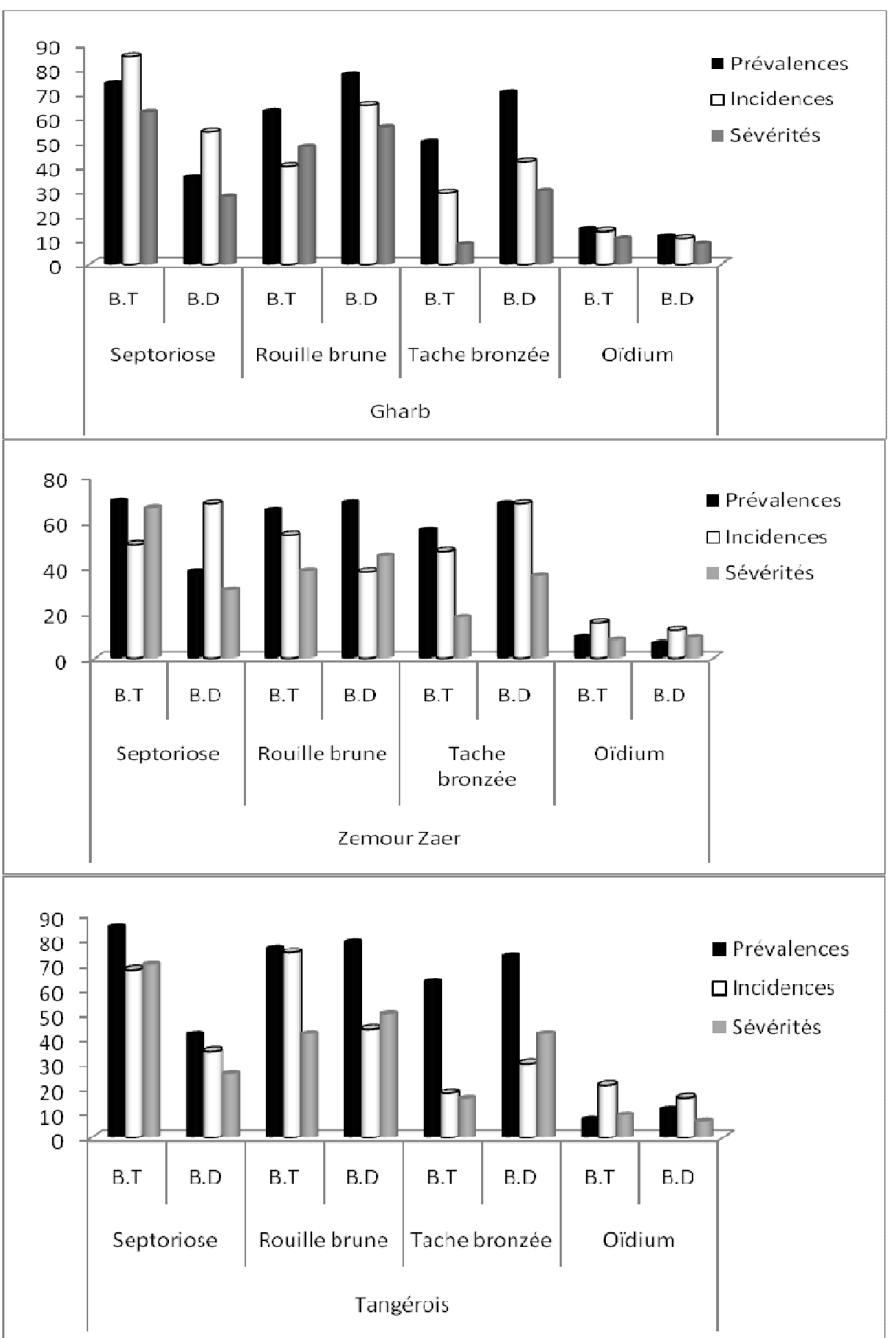

Fig. 5 : Prévalences, incidences et sévérités (\%) des principales maladies cryptogamiques foliaires du blé dans trois régions céréalières du Maroc lors de la campagne 2012-2013. 

Maroc en 2013

La présente étude a révélé que parmi les deux espèces de Septoria identifiées, Septoria tritici est la plus abondante et la plus sévère et continue à dominer, alors que Stagonospora nodorum reste toujours limité. En effet, des échantillons de feuilles de blé attaqués par les septorioses ont été examinés et ont montré que parmi
700 pycnides observées sous microscope, $71 \%$ étaient celles de S. tritici, et $29 \%$ étaient celles de Stagonospora nodorum. La répartition des fréquences des deux espèces de Septoria par région est donnée dans le tableau 3.

Tableau 3. Fréquences (\%) des deux espèces de Septoria au niveau des trois régions céréalières du Maroc en 20122013.

\begin{tabular}{l|c|c|c}
\hline & \multicolumn{3}{|c}{ Nombre d'échantillons (fréquence \%) par régions } \\
\hline Septoria spp. & Gharb & Zemour - Zaer & Tangérois \\
\hline Septoria tritici & $168(72 \%)$ & $158(68 \%)$ & $173(74 \%)$ \\
\hline Stagonospora nodorum & $65(28 \%)$ & $75(32 \%)$ & $61(26 \%)$ \\
\hline
\end{tabular}

\section{DISCUSSION ET CONCLUSION}

Durant la campagne agricole 2012-2013, la pluviométrie était importante ( $P>500 \mathrm{~mm}$ ) (Balaghi et al., 2013) et bien répartie dans le temps et dans l'espace dans la majorité des régions céréalières et potentiellement dans les régions du Nord-ouest du Maroc. Ceci a bien favorisé le développement des maladies cryptogamiques foliaires du blé. Cette situation inquiétante a suscité l'intervention de l'état par un programme de traitements fongicides contre les maladies cryptogamiques des céréales dans les principales régions céréalières du royaume, afin de minimiser leur impact sur le rendement. Les septorioses se sont bien développées, surtout sur blé tendre, atteignant des sévérités moyennes de $70 \%$ de tissu foliaire attaqué. Au niveau de certaines parcelles, des séverités de $90 \%$ ont été notées. Ceci confirme les résultats des prospections antérieures (Lyamani, 1990; Mazouz et al., 1995; Farih et Ezzahiri, 1996) et montre que les septorioses sont les maladies les plus importantes sur blé tendre pendant les années pluvieuses. La variation des sévérités des deux espèces de Septoria d'une région à l'autre peut s'expliquer par les différences des conditions climatiques (Shaner, 1981), de la population de l'agent pathogène (Eyal et al., 1987) et les variétés cultivées (Shaner, 1981; Gough et Smith, 1985). Au Maroc, Saadaoui, (1975) a rapporté depuis 1975 l'existence de deux espèces de Septoria : Septoria tritici et Stagonospora nodorum et a trouvé que $S$. tritici est l'espèce prédominante. Cette situation est restée inchangée dans cette étude. Cependant, cette tendance a changé durant la campagne agricole 95/96 avec une prédominance de $S$. nodorum quelque soit l'espèce de blé cultivée. S. nodorum était plus fréquente $(37,5 \%)$ sur le blé tendre que S. tritici (25\%) (Farih et Ezzahiri, 1996). La distribution des fréquences des deux espèces de Septoria a été beaucoup influencée par les conditions climatiques, l'espèce du blé cultivée, et la localisation géographique de la culture (Farih et Ezzahiri, 1996). En Europe, Stagonospora nodorum est l'espèce principale. Eyal et al., (1987) a expliqué son importance secondaire en Afrique du nord par une exigence plus prononcée du champignon en humidité. Au Maroc, la sous estimation du champignon est favorisée par sa faible production de pycnides et ses symptômes facilement confondus avec ceux d'autres maladies foliaires, en particulier Pyrenophora tritici repentis (Mazouz et al., 1995). L'importance des rouilles brunes était évidente vue leur distribution régulière dans les trois régions prospectées, avec des prévalences plus ou moins similaires sur les deux espèces du blé. La tache bronzée s'est montrée plus sévère sur le blé dur dans toutes les régions prospectées (42\% au Tangérois), ce qui peut être expliqué par la sensibilité des variétés cultivées à cette maladie. L'oïdium était le moins prévalent et le moins sévère dans cette campagne agricole, même si les conditions climatiques étaient propices à son développement. Ses sévérités faibles sont dues essentiellement à son apparition plus tardivement dans le cycle de la culture du blé, en plus de la concurrence par les autres maladies. Le développement de la rouille jaune est limité aux plaines intérieures au piémont de l'Atlas au Maroc (Tadla, le Tassaout et le Saïs) (Sayoud et al., 1999; Ezzahiri, 2001), ce qui explique l'absence de la maladie dans les trois régions prospectées. La rouille noire historiquement importante, n'a pas été révélée dans les trois régions prospectées grâce également à son apparition tardive et à la précocité et la résistance de la plupart des variétés du blé cultivées (Sayoud et al., 1999; Ezzahiri, 2001). La dominance des emblavures de blé tendre au Maroc ( 2 millions d'ha de blé tendre et 1 million de blé dur) pourrait amoindrir les prévalences de 
certaines maladies sur le blé dur, c'est le cas des septorioses (Stagonospora nodorum). En examinant la figure 5 , les prévalences élevées des maladies

\section{RÉFÉRENCES}

Balaghi R., Tahri M., El Hairech T., 2013. Bulletin de suivi agro météorologique de la campagne céréalière de 2012-2013. Crop Growth Monitoring System - MAROC, $4 \mathrm{p}$.

Boulif M., 2011. Gestion intégrée des maladies du blé. Documentation d'appui. ENA de Meknès, 12p.

Eyal Z., Sharen A.L., Prescot J.M., et Van Ginkel M., 1987. The Septoria diseases of wheat: concepts and methods of disease management. Mexico, D. F: CIMMYT, $52 \mathrm{p}$.

Ezzahiri B., 2001. Les maladies du blé : identification, facteurs de développement et méthodes de lutte. Bulletin de transfert de technologie en agriculture, $\mathrm{N}^{\circ} 77,4 \mathrm{p}$.

Farih A., Ezzahiri B., 1996. Distribution et importance des septorioses au Maroc. Proc. Symp. Rég. Les maladies des céréales et des légumineuses alimentaires, 11-14 November 1996, Rabat, Maroc, Ezzahiri B., Al Yamani A., Farih A., El Yamani M., (Eds.), Rabat, INRA, 390 p.

Gough F.J., et Smith E.L., 1985. A genetic analysis of Triticum aestivum 'Vilmorin' resistance to speckled leaf blotch and Pyrenophora tan spot, 36p. In Scharen A.L. (ed.). Septoria of cereals: Proceeding of the workshop, 1983. Montana State University, Bozeman.

Lyamani A., 1990. Fungal foliar diseases of wheat and barley in Morocco, pp 281-282. In 8 th Congress of the Mediterranean Phytopathological Union. October 28 th, November 3th 1990. Agadir, Morocco.

Mazouz H., Saadaoui E.M., Jlibene M., et Lyamani A., 1995. Importance de la septoriose du blé au Maroc en 1991. Al Awamia, 91: 63-69.

Saadaoui E.M., 1975. Contribution à l'étude des septorioses du blé au Maroc. Mém. 3ème Cycle, Inst. Agro. Vét. Hassan II, Rabat, Maroc, $40 \mathrm{p}$.

Sayoud R., Ezzahiri B., et Bouznad Z., 1999. Les maladies des céréales et des légumineuses alimentaires au Maghreb. ITGC, Alger. Guide pratique, 64p.

Shanner G., Finney R.E., et Patterson F.L., 1975. Expression and effectiveness of resistance in wheat to Septoria leaf blotch. Phytopathology, 65: 761-766. accompagnées par des incidences plus faibles, permet de supposer que l'inoculum primaire est de nature exogène.

Shaner G., 1981. Effect of environment on fungal leaf blights of small grains. Annu. Rev. Phytopathology, 19: 273-296.

Zillinsky F.J., 1983. Maladies communes des céréales à paille. Guide d'identification. Mexico, D.F CIMMYT, $141 \mathrm{p}$. 